\title{
BROADBAND INDOOR WIRELESS SYSTEM WITH IN-CELL FREQUENCY REUSE BASED ON SECTORED ANTENNAS *
}

\author{
Aleandro S. Macedo and Elvino S. Sousa \\ Dept. of Electrical and Computer Engineering \\ University of Toronto \\ Toronto, Ontario M5S 3G4, Canada \\ Phones: +1-416-978-3651, Fax: +1-416-978-4425 \\ E-mails: (macedo, sousa)@comm.toronto.edu
}

\begin{abstract}
Resumo : Neste artigo investiga-se um novo sistema de múltiplo acesso para comunicações sem fio de banda larga em interiores utilizando antenas setorizadas. $O$ que o difere de outros sistemas previamente propostos, que também utilizam antenas setorizadas, é a sua capacidade de reusar frequências em setores distintos da microcélula : terminais portáteis localizados em setores distintos da microcélula podem compartilhar o mesmo espectro de frequência se o nível de interferência mútua for aceitável. Porém, isto requer uma cuidadosa programação das transmissões a fim de evitar interferências destrutivas. A programação ótima, a que maximiza o número de pacotes transmitidos por quadro, se encontra na classe de problemas NP-completos para o caso de três ou mais transmissões simultâneas. Como este tipo de problema não pode ser resolvido em tempo real, propõe-se um algoritmo subótimo chamado "First Fit Algorithm". O problema é estudado experimentalmente para o caso em que a microcélula é dividida em dez setores (setorização de nível dez). Um modelo estatístico de propagação por caminhos múltiplos é utilizado para estender os resultados para outros níveis de setorização. O sistema proposto pode proporcionar um grande incremento na capacidade da microcélula. Por exemplo, enquanto os sistemas previamente propostos podem transmitir somente um pacote de informação por vez, este novo sistema é capaz de transmitir, em média, perto de três pacotes simultaneamente. Este resultado é válido para um nível de interferência mútua máximo de $10 \mathrm{~dB}$ com setorização de nível dez em um ambiente interno com paredes divisórias feitas com blocos de concreto.
\end{abstract}

Abstract: This paper investigates a new multiple access system for broadband indoor wireless communications based on sectored antennas. Unlike previous sectored antenna indoor systems, this system incorporates the capability of reusing spectrum in different antenna sectors of the base station. It is shown that portable terminals located in different sectors of the indoor microcell may transmit/receive simultaneous data packets on the same frequency if their mutual interference is below a threshold level (or capture threshold) that guarantees an acceptable packet error rate. However, this requires careful scheduling of packet transmissions in order to avoid transmitting packets that would jam each other during the same time slot. When trying to transmit more than two packets per time slot, the optimum scheduling problem, the one that maximizes the number of packets transmitted per

\footnotetext{
* This work was supported in part by the Brazilian Council for Scientific and Technological Research (CNPq), and in part by Bell Mobility Cellular.
}

frame, is in the NP-complete class of problems, so it can not be solved in real time. Therefore, a sub-optimum algorithm named First Fit Algorighm is proposed for the frame scheduling problem. The problem is investigated experimentally for a sectorization level of ten (using ten antenna sectors in the base station). A statistical model of multipath propagation is used to extend the results to other levels of sectorization. The proposed system can significantly increase capacity when compared with systems that can transmit a single data packet per time slot. For example, while previous systems can transmit only one packet at a time, the proposed system can transmit on average close to three packets per time slot when operating with a capture threshold of $10 \mathrm{~dB}$ and ten antenna sectors in a closed indoor location with internal walls of concrete blocks.

Keywords: Indoor Wireless Communications, Frequency Reuse, Sectored Antennas.

\section{INTRODUCTION}

Sectored Antennas have been used for some time in mobile cellular systems as a means of reducing interference and consequently increasing system capacity. More recently, sectored antennas have also been applied to Wireless Local Area Networks (WLAN) [1] [2]. In [1] the use of directional antennas is considered to be an excellent technique for dealing with multipath propagation problems. In [2] sectored antennas are proposed in order to combat the severe co-channel interference arising from a small frequency reuse factor. Although [1] and [2] propose different rules for the use of sectored antennas, the final goal in both cases is to achieve an increase in system capacity.

The multiple access schemes described in [1] and [2] allow transmission/reception in only one of the antenna sectors at a time, usually in the one that provides the best communication channel between the base station and a given portable. Figure 1-a shows sectored antennas similar to those in [1] and [2]. The RF switch matrix used to select the desired antenna from the set can be fabricated compactly and inexpensively using either a Gallium Arsenide Monolithic Microwave Integrated Circuit (GaAs MMIC) or microwave diode switching elements [4].

In [3], a new multiple-access scheme is proposed to achieve in-cell frequency reuse by allowing simultaneous transmissions over different antenna sectors. This new 
multiple-access scheme requires a multi-port sectored antenna system as the one illustrated in Figure 1-b, which represents the same set of antennas as in Figure 1-a but with a switch matrix capable of simultaneously selecting two antennas and connecting each to a desired port that is connected to a transceiver. The sectored antenna system of Figure 1-b has the potential to double the capacity of the system by allowing simultaneous transmissions/receptions to occur in two antenna sectors sharing the same frequency spectrum. However, as we explain in Section 2, this kind of spectrum sharing requires compatible portables. (a)

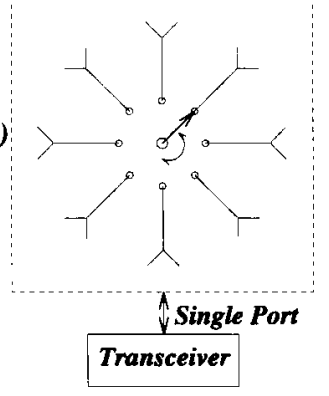

(b)

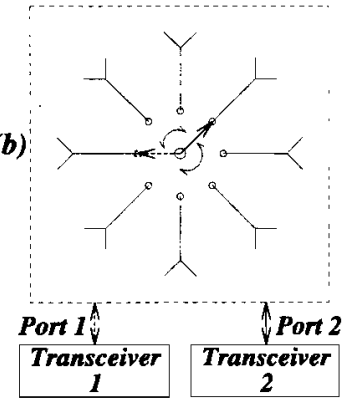

Figure 1: (a) Sectored antenna system similar to those in [1] and [2]; (b) The 2-port sectored antenna system.

The schemes proposed in [1] and [2] employ sectored antennas in both the base station and portable modules. The scheme proposed in [3] assumes that this approach causes the system to become excessively complex, and, due to the size of the sectored antenna system, not suitable for a portable terminal. Therefore the conservative approach of concentrating the complexity of the system in the base station is adopted in order to be able to use simpler antennas in the portables: sectored antennas are used in the base station and simple omnidirectional antennas are used in the portables.

The results presented in [3] are based on measurement data obtained with a sectorization level of 10 (using 10 sectors in the base station). In this paper we use statistical modeling of indoor multipath propagation in order to investigate the performance of the multiple access proposed in [3] when operating with different levels of sectorization.

\section{COMPATIBILITY CONDITION}

The concept of compatibility is explained with the help of Figure 2 which shows a base station using a 2-port sectored antenna system to communicate simultaneously with two portables, $\mathbf{P}_{1}$ and $\mathbf{P}_{2}$. The signal strength values quoted in this figure were chosen for illustrative purposes. The reflectors represent reflecting structures, for example, walls or metallic doors. In order to verify the compatibility between portables $\mathbf{P}_{1}$ and $\mathbf{P}_{2}$, the minimum Signal-to-Interference Ratio (SIR) acceptable for communication between the base station and a portable has to be specified. This parameter is the capture threshold, which we name $\Pi$. Its value is dependent on the coding and on the modulation scheme employed. For example, spread spectrum modulation schemes can operate with a level of interference above the level of the signal, 72 which results in a negative value of $\Pi$ (in decibel). However, the proposed multiple access scheme is intended to operate with narrow band (non spread spectrum) modulation schemes which can achieve high spectrum efficiency, around $1 \mathrm{bps} / \mathrm{Hz}$, as required in the FCC proposal for U-NII wireless networks [5]. Therefore we will be considering values of $\Pi$ in the range of 5 to $20 \mathrm{~dB}$.

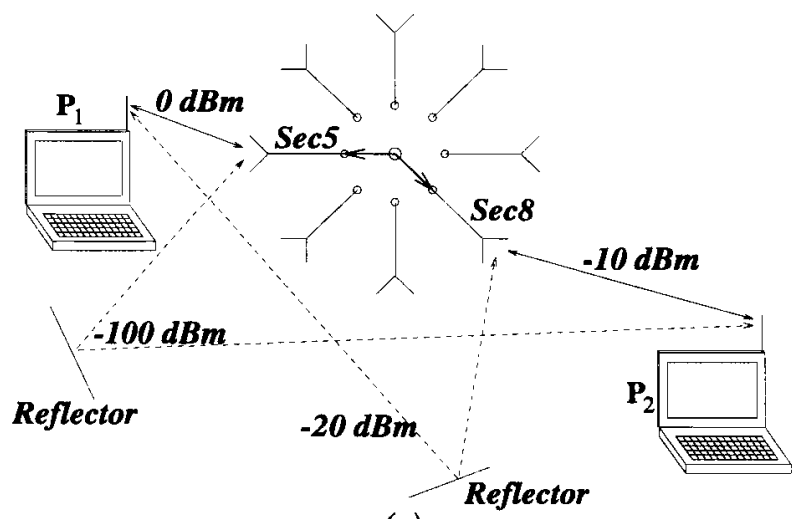

(a)

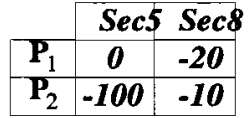

(b)

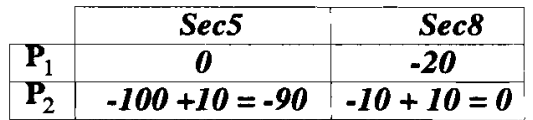

(c)
Figure 2: (a) A base station communicating simultaneously with two portables; (b) Table of average power levels (dBm) received without power control; (c) Table of average power levels $(\mathrm{dBm})$ received with power control.

It is assumed that an up-link transmission and a down-link transmission can not occur simultaneously: either both transmissions are in the up-link direction (from portable to base station) or both are in the down-link direction (from base station to portable). This assumption is made because the level of interference that a down-link transmission would cause in an up-link reception would be unacceptable: in the base station, the power level of a transmitted signal is much higher than the power level of a received signal.

In the down-link case, the base station uses antenna sector 5 to transmit to portable $\mathbf{P}_{1}$, and antenna sector 8 to transmit to portable $\mathbf{P}_{2}$. Portable $\mathbf{P}_{1}$ receives a signal from antenna sector 5 at an average power level of $0 \mathrm{dBm}$ and interference from antenna sector 8 through a reflected path at an average power level of $-20 \mathrm{dBm}$. Simultaneously portable $\mathbf{P}_{2}$ receives a signal from antenna sector 8 at an average power level of $-10 \mathrm{dBm}$ and interference from antenna sector 5 through a reflected path at an average power level of $-100 \mathrm{dBm}$. Therefore, the SIR for the signal received by portable $\mathbf{P}_{1}$ is $20 \mathrm{~dB}$, and the SIR for the signal received by portable $\mathbf{P}_{2}$ is $90 \mathrm{~dB}$. Then we say that portables $\mathbf{P}_{1}$ and $\mathbf{P}_{2}$ are compatible in the down-link case if $\Pi \leq 20 \mathrm{~dB}$.

In the up-link case, the base station receives, through antenna sector 5 , the signal transmitted by $\mathbf{P}_{1}$ at an average power level of $0 \mathrm{dBm}$ and interference of $-100 \mathrm{dBm}$ from the reflected signal transmitted by $P_{2}$, yielding an SIR of $100 \mathrm{~dB}$ for the $\mathbf{P}_{1}$ transmitted signal. Simultaneously, the base station receives, through antenna sector 8 , the signal transmitted by 
$\mathbf{P}_{2}$ at an average power level of $-10 \mathrm{dBm}$ and interference of $-20 \mathrm{dBm}$ from the reflected signal transmitted by $\mathbf{P}_{1}$, yielding an SIR of $10 \mathrm{~dB}$ for the $\mathbf{P}_{2}$ transmitted signal. Therefore we say that portables $\mathbf{P}_{1}$ and $\mathbf{P}_{2}$ are compatible in the up-link case if $\Pi \leq 10 \mathrm{~dB}$.

In this example, which does not consider the power control mechanism that we describe below, compatibility in the down-link case does not necessarily correspond to compatibility in the up-link case. In fact, we observed through measurement results that the average number of compatibilities among portables in an indoor microcell is, in general, larger for the down-link case than for the up-link case. This downlink/up-link average compatibility asymmetry arises because, in the up-link case, portables located closer to the base station cause stronger interference with the signals of portables located on the periphery of the indoor microcell. In a sense this problem is similar to the near-far effect that decreases the up-link capacity of CDMA systems. Therefore it is expected that power control can be used to improve the average number of compatibilities in the up-link case.

Consider now the use of power control in Figure 2. The power control mechanism assumes that the portables adjust their transmitting power so that the base station receives, through the best sector for communication with a given portable, the same average power level from any portable. In this case, portable $\mathbf{P}_{2}$ increases its transmitted power so that the base station can receive its signal with the same power level of the signal received from portable $\mathbf{P}_{1}$. Then $\mathbf{P}_{2}$ has to add $10 \mathrm{~dB}$ to its transmitted signal power. By doing so, it also adds $10 \mathrm{~dB}$ to the interference caused in the $\mathbf{P}_{1}$ signal. This is shown in figure $2-c$. If the up-link SIR values are recalculated with the power control values of figure 2-c, the SIR values obtained are $90 \mathrm{~dB}$ for the $\mathbf{P}_{1}$ signal and $20 \mathrm{~dB}$ for the $\mathbf{P}_{2}$ signal. Therefore, the minimum of the two SIR values, $20 \mathrm{~dB}$, is $10 \mathrm{~dB}$ better than the minimum SIR value obtained without power control.

In order to express the compatibility conditions mathematically we define the following parameters:

- $G_{p, s}$ (Portable-Sector Power Gain): represents the average power received through the channel between portable $\mathbf{P}_{p}$ and antenna sector $s$. It has the same value for both up-link and down-link channels (provided that the same power level is transmitted in both cases). This is true because we assume that up-link and down-link transmissions occur in the same frequency band (but not at the same time); therefore up-link and down-link channels are reciprocal.

- $B(i)$ : represents the best antenna sector for communicating with $\mathbf{P}_{i}$.

The $G_{p, s}$ parameters are illustrated in figure 3 .

Using these definitions, we can express the conditions for the existence of compatibility in the down-link case between two portables $\mathbf{P}_{i}$ and $\mathbf{P}_{j}$ as:

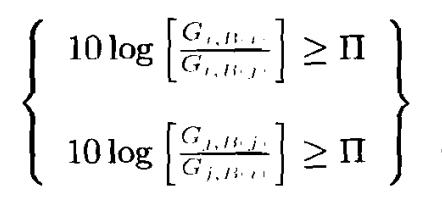

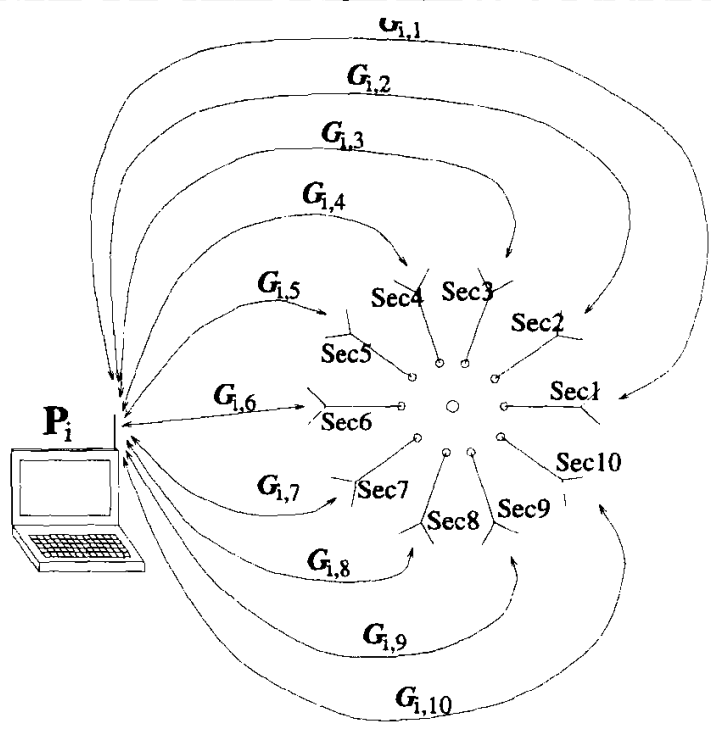

Figure 3: Illustration of the $G_{p, s}$ parameter.

In the up-link case the conditions for the existence of compatibility between two portables $\mathbf{P}_{i}$ and $\mathbf{P}_{j}$ are:

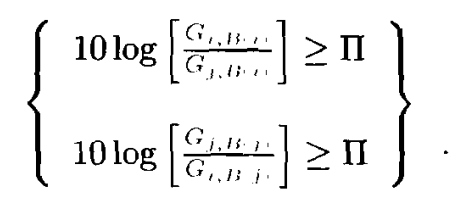

\section{COMPATIBILITY CONDITION FOR THE N-PORT CASE}

In the previous section, we considered compatibility for the 2-port case, which implies using a system of sectored antennas with two ports as shown in figure 1-b. Now we consider a system of sectored antennas with $N$ ports as shown in figure 4 .

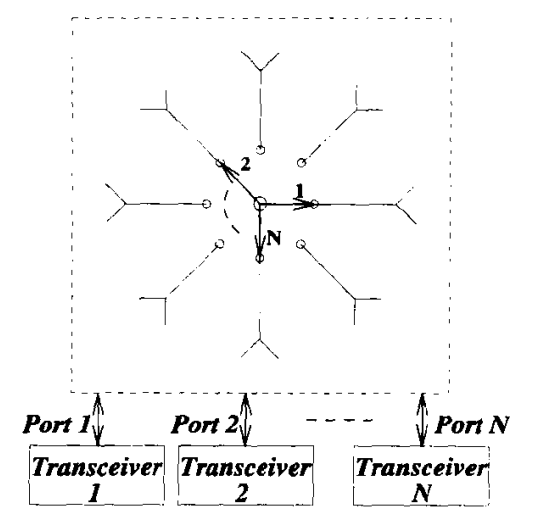

Figure 4: The N-port sectored antenna system.

An $N$-port antenna system would allow a maximum of $N$ simultaneous transmissions in $N$ different antenna sectors. As such it would have a switching matrix capable of selecting $N$ of the antenna sectors and connecting each of them to one of $N$ ports (each port is connected to a transceiver). In this 
case the conditions for compatibility among $N$ portables $\mathbf{P}_{1}$, $\mathbf{P}_{2}, \ldots, \mathbf{P}_{N}$ in the down-link case are given in (3).

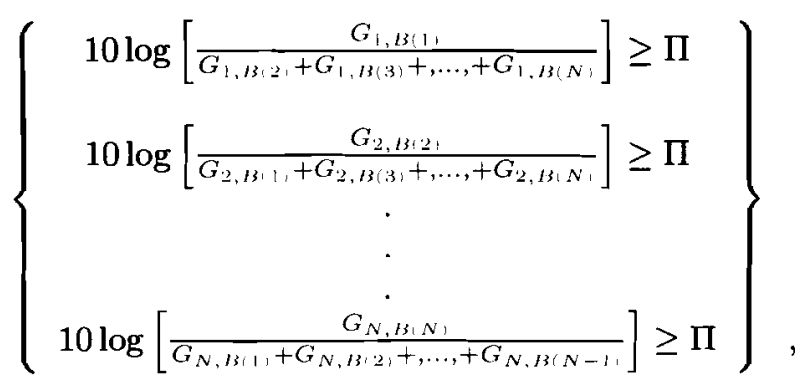

and, in the up-link case, the compatibility conditions are given in (4). Note that these $N$ portables represent a subset of all the portables in the microcell.

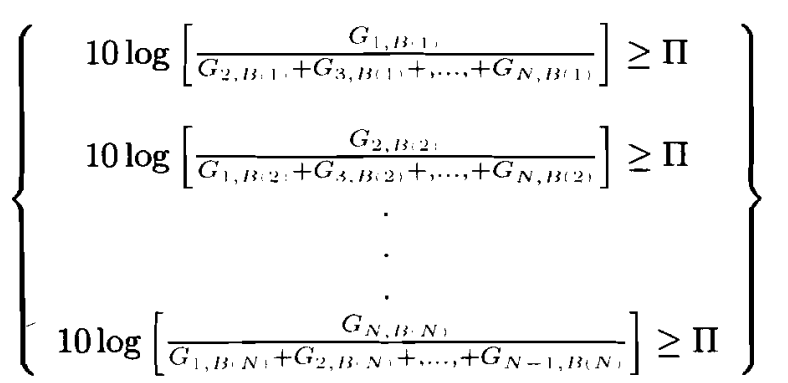

If the $N$ conditions of (3) are satisfied, then there may be $N$ simultaneous packet transmissions during a time slot to these $N$ portables (down-link case). This means that, during this time slot, the down-link system capacity is multiplied by $N$, i.e., $N$ packets are transmitted through $N$ sectors instead of the single packet that could be transmitted in this time slot if a single port sectored antenna system were used in the base station. Similarly, if the $N$ conditions of (4) are satisfied, then there may be $N$ simultaneous packet transmissions during a time slot from these $N$ portables (up-link case), which corresponds to the up-link system capacity being multiplied by $N$. In general, however, an increase in the number of ports does not correspond to a proportional increase in system capacity, as can be observed from measurement and simulation results presented later in this paper. The reason is that the number of subsets of $N$ portables that correspond to compatible portables decrease as $N$ increases. For example, the probability of two portables being compatible is larger than the probability of three portables being compatible. Therefore, as the number of ports $N$ increase, it is more likely that some of them will not be used during a given time slot due to a lack of compatible portables. The capacity gain eventually saturates at a given number of ports.

\section{CHANNEL MEASUREMENTS}

We devised an experiment which allowed us to measure the portable-sector power gain $\left(G_{p, s}\right.$, see Figure 3 ) values for a base station and portables located in some typical indoor environments. With these values, we were able to verify the compatibility among portables using the conditions established in (3) or (4). The experimental setup is described with details in [3]. It can measure with a sectorization level of ten, i.e., it assumes that the base station operates with ten antenna sectors, each covering $36^{\circ}$ of the horizontal plane. We measured different indoor locations using this experimental setup. One of them, which we chose to investigate in this paper, is the southwest section of the fourth floor of the Galbraith Building located in the University of Toronto Campus. This location is depicted in Figure 5, and we named it location 1. It represents a closed indoor environment where the walls are made of a hard material, in this case concrete blocks. For this location, 50 uniformly distributed positions were measured to simulate 50 portables.

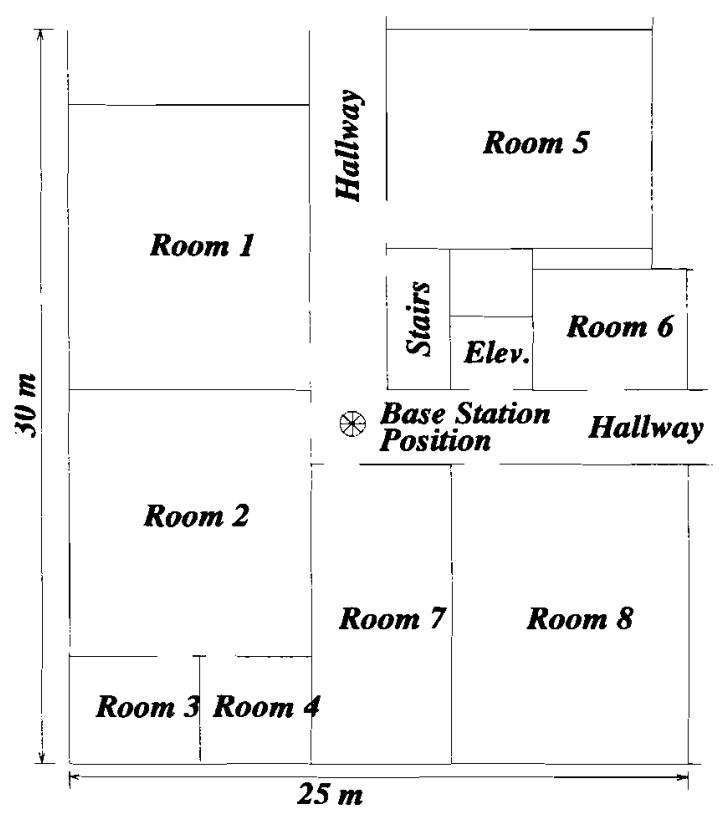

Figure 5: Indoor Location 1.

\section{MEASUREMENT RESULTS}

In this section, we present the measurement results in terms of average compatibilities which we define as follows.

The average compatibility between two portables is given by

$$
A C 2=\frac{1}{\left(\begin{array}{c}
N_{p} \\
2
\end{array}\right)} \sum_{i=1}^{N_{j}} \sum_{j=i+1}^{N_{i}} I(i, j)
$$

where $N_{p}$ is the number of portables in the microcell, and $I(i, j)$ is defined for portable $i$ and $j$ as

$$
I(i, j)= \begin{cases}1, & \begin{array}{l}
\text { if portables } i \text { and } j \text { satisfy } \\
\text { compatibility condition. }
\end{array} \\
0, & \text { otherwise. }\end{cases}
$$

Here, $A C 2$ represents the fraction of all two portable combinations that correspond to compatible pairs. Therefore it represents the probability of two randomly picked portables being compatible. 

by

The average compatibility among three portables is given

$$
A C 3=\frac{1}{\left(\begin{array}{c}
N_{p} \\
3
\end{array}\right)} \sum_{i=1}^{N_{p}} \sum_{j=i+1}^{N_{j}} \sum_{k=j+1}^{N_{p}} I(i, j, k),
$$

where $I(i, j, k)$ is defined for portable $i, j$ and $k$ as

$$
I(i, j, k)= \begin{cases}1, & \begin{array}{l}
\text { if portables } i, j \text { and } k \text { satisfy } \\
\text { compatibility condition. }
\end{array} \\
0, & \text { otherwise. }\end{cases}
$$

Here, $A C 3$ represents the fraction of all three portable combinations that correspond to compatible triples. Therefore it represents the probability of three randomly picked portables being compatible.

Similarly, $A C n$ represents the fraction of all $n$ portable combinations that correspond to compatible subsets of $n$ portables.

Figure 6 shows the results obtained in the up-link case for the location 1. The average compatibilities $A C 2, A C 3$ and $A C 4$ are plotted as functions of the capture threshold. The solid curves are the results obtained considering the power control mechanism described in Section 2. Figure 7 shows the average compatibilities obtained in the down-link case for location 1. In the down-link case, assuming that the same power level is transmitted to all the portables, we obtained average compatibilities close to those obtained in the up-link case with power control.

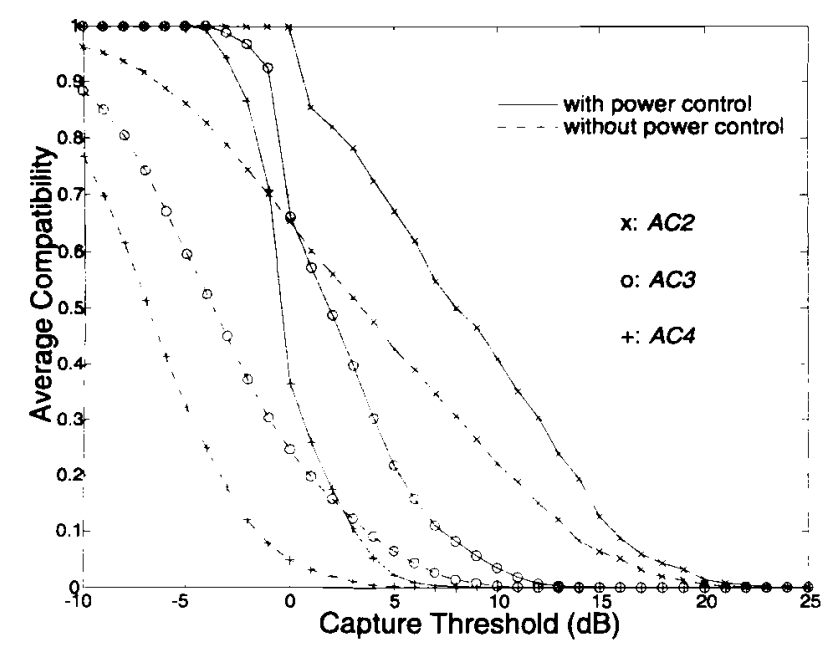

Figure 6: Up-link average compatibilities in location 1.

In order to understand the meaning of these measurement results, let us assume a multiple access scheme in which a base station operating with a sectored antenna system like that shown in figure 4 allocates packets in time slots in order to transmit them to portables in the microcell. Let us also assume that there is no compatibility verification in this process, i.e, packets are picked randomly. Therefore, when two or more packets are transmitted in the same time slot, they may be received with errors if the receiving portables are not compatible, and in this case we say that a collision occurs. The probability that a time slot is collision-free depends

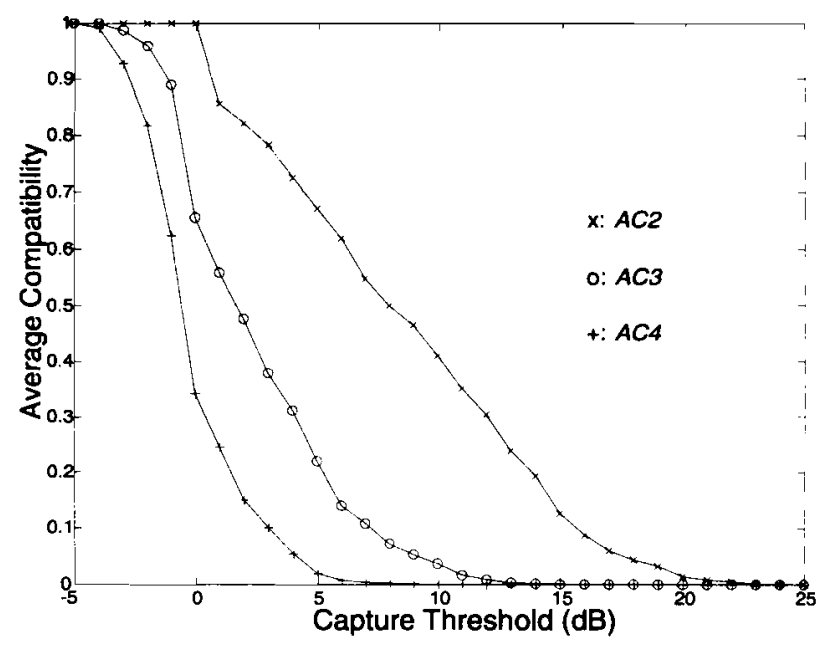

Figure 7: Down-link average compatibilities in location 1.

on the number of packets transmitted in this time slot. This probability is the average compatibility among the portables. Consider for example a capture threshold of $10 \mathrm{~dB}$ in Figure 7. In this case the probability of a collision-free time slot is approximately 0.4 if two packets are transmitted together, and approximately 0.04 if three portables are transmitted together. We may conclude that such a multiple access scheme would provide poor throughput due to the small likelihood of collision-free transmissions. A new multiple access scheme that verifies compatibility among the portables in order to avoid simultaneously transmitting packets that can jam each other is proposed in [3]. Such a scheme can significantly improve the throughput when compared with the one-port case described in [1] and [2]. This new multiple access scheme is described in the following section.

\section{FRAME SCHEDULING}

This section discusses how to take advantage of the existing compatibilities among portables in an indoor microcell in order to increase system capacity. This can be done by properly scheduling simultaneous transmissions of packets that belong to compatible portables. Figure 8 depicts the scenario where scheduling is performed. It shows that the base station has a buffer where packets collected from a high speed wireline backbone network are temporarily stored while waiting to be transmitted to their destination portables during down-link time slots. It also shows that each portable has a buffer that temporarily stores packets generated in the end-user terminal equipment and are waiting to be transmitted to the base station during granted up-link time slots. The scheduling problem consists in finding among the awaiting packets those that can share a time slot in the next frame, namely, those that belong to compatible portables.

Let us suppose that Figure 9-a represents the values of the $G_{p, s}$ parameters, as defined in Section 2, related to the portables and the base station of figure 8 . The values quoted in this figure were chosen for illustrative purposes. With these values, the two by two compatibility relations among the 


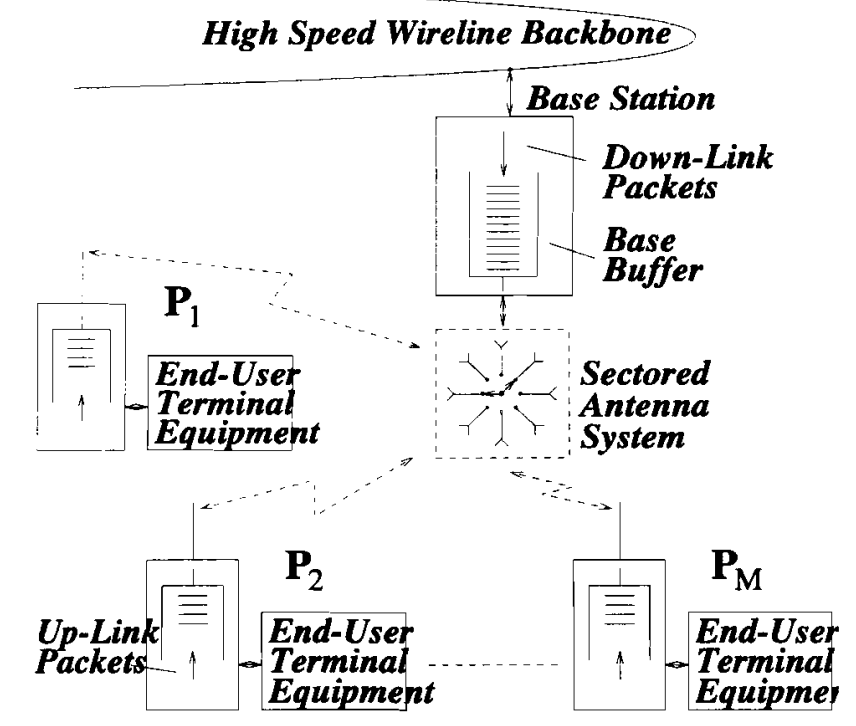

Figure 8: A microcell with its base station and portables.

portables can be obtained, as shown in figure 9-b for a capture threshold of $10 \mathrm{~dB}$, where " 1 " means compatibility and " 0 " means incompatibility. Let us also suppose that a 2-port frame has been scheduled using these compatibility relations. figure 10 represents this frame with its down-link and up-link portions. Here, the word "port" is used to denote a port in the sectored antenna system which can be switched to any one of the antenna sectors. Therefore, in this figure, the base station operates with a two-port sectored antenna system as shown in figure 1-b. The frame is composed of a number of equal time slots, each capable of carrying one packet per port. Thus, with the two-port antenna system, a maximum of two packets can be transmitted per time slot. For example, each packet could transport one ATM (Asynchronous Transfer Mode) cell [7] plus some wireless overhead. The packet owners and the sectors being used for the transmission of each packet are also shown in figure 10 . The first time slot of the down-link subframe carries two packets, one for $\mathbf{P}_{1}$ and the other for $\mathbf{P}_{3}$. For each packet, the base station uses the antenna sectors that provide the best signal level, namely, sectors 2 and 6 . The time slot and frequency spectrum sharing between a $\mathbf{P}_{1}$ packet and a $\mathbf{P}_{3}$ packet is possible because of the compatibility between their owners.

We propose that up-link and down-link modes use the same frequency spectrum (Time Division Duplex). This guarantees reciprocity between up-link and down-link channels, allowing the $G_{p, s}$ parameters to be used for both down-link and up-link compatibility verification with the conditions defined in (1) and (2), respectively. Up-link/down-link traffic does not need to be symmetrical. In fact it is expected that the up-link traffic will be a small fraction of the down-link traffic. This is the case, for example, for the current traffic in the Internet where the down-loading of files generates highly asymmetrical traffic. Accordingly, the down-link subframe in figure 10 was made intentionally larger than the up-link subframe for illustrative purposes.

It is assumed that scheduling is performed in the base station (central control architecture). For this purpose the base
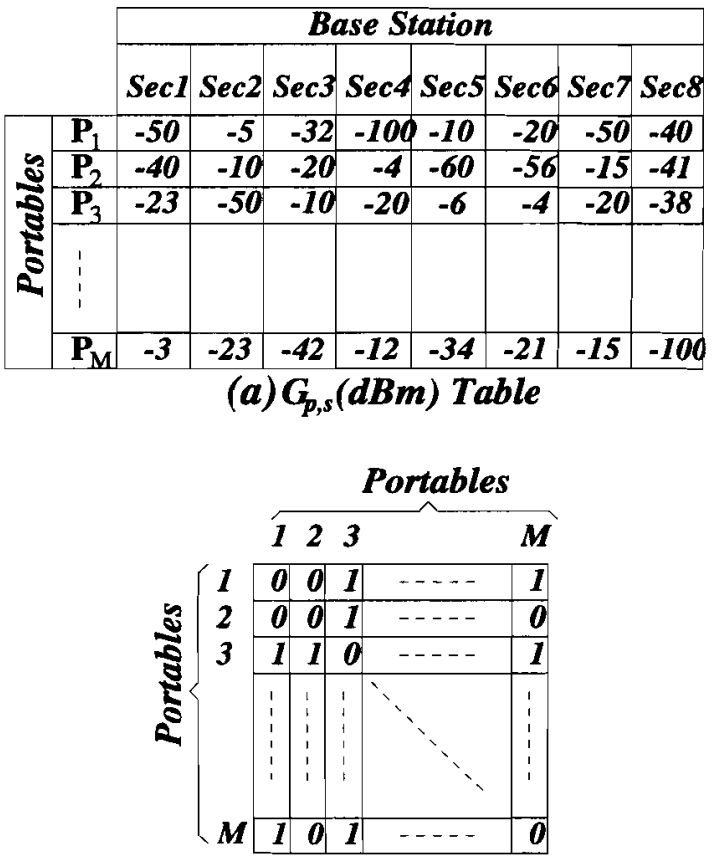

(b) Compatibility Matrix

Figure 9: (a) $G_{p, s}$ parameters; and (b) Matrix of compatibility of pairs of portables in the microcell in figure 8 .

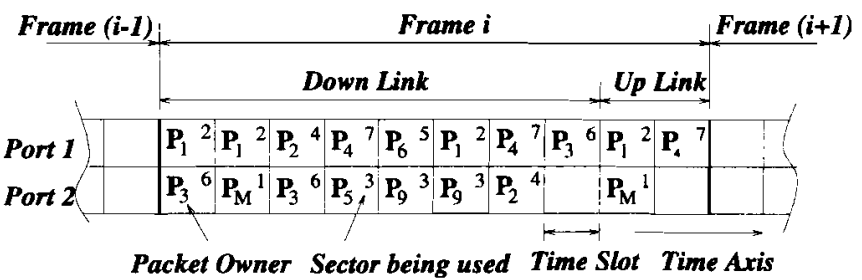

Figure 10: A two-port frame schedule.

receives updated information about the compatibility relations among the portables and about the number of packets waiting transmission in each buffer. For Variable Bit Rate (VBR) services, the number of packets that arrive in the portables' buffers and in the base station's buffer during one frame duration varies from frame to frame. This means that each new frame requires its own scheduling. Therefore the time available for scheduling each frame is one frame duration: during the transmission of the $i$-th frame, the base station is working out the scheduling of the $(i+1)$-th frame, which has to be ready for transmission before the end of the $i$-th frame transmission.

\section{THE FIRST FIT ALGORITHM}

The necessity of solving the scheduling problem in less than one frame duration was stated in the previous section. However, it turns out that for the $N$-port case $(N \geq 3$ ), the optimum scheduling problem, which maximize the number of packets transmitted per frame, is in the NP-Complete class of problems (problems that can not be solved in polynomial time, or Non Polynomial problems) [6]. The practical result 
is that, for a large number of packets, solving it in real time becomes impractical.

The NP-Completeness of this problem is not proved here, but it is indicated that it can be proved by mapping the $\mathrm{N}$ Dimensional Matching problem [6] to the $N$-channel $(N \geq$ 3) frame scheduling problem. By knowing that the $\mathrm{N}$ Dimensional ( $N \geq 3$ ) Matching problem is in the class of NP-Complete problems, it is established that the $N$-channel frame scheduling problem $(N \geq 3)$ is also in this class. The 2-port optimum frame scheduling problem is a special case that requires further investigation to determine if it belongs to the NP-complete class or if it belongs to the $\mathbf{P}$ class (problems that can be solved in polynomial time [6]). However we prefer to concentrate on finding a sub-optimum frame scheduling solution that fits the generic $N$-port case, and that can be solved in real time. This sub-optimum solution is the First Fit Algorithm (FFA) that we describe below.

The FFA can be better explained with the help of figure 11 . This algorithm places the packets into the time slots one at a time in the order that they arrive in the buffer. It does so according to the following simple rules:

1. First, the algorithm occupies the time slots of the first port. If, after performing this step, there are packets left in the buffer, then it performs the following steps.

2. For allocating packets in ports 2 and up, the algorithm always places the next packet of the buffer into the lowestindexed time slot containing only packets that are compatible with the packet being allocated. The conditions of compatibility are given by (3) for the down-link case and by (4) for the up-link case, where $N$ is such that $N-1$ is the number of packets already allocated in the time slot. When searching for the lowest-indexed time slot, the port index has priority over the time slot index. Therefore the algorithm looks first for a time slot in port 2 , and if it can not find a valid time slot, then it looks for one in port 3 , and so on. This helps to distribute packet transmissions evenly in the time axis.

3. If a packet does not fit in any of the time slots of this frame, then the algorithm leaves it to be transported in the next frame and tries to allocate the next packet of the buffer by going back to step 2 .

4. The algorithm repeats steps 2 and 3 until all the packets of the buffer are considered or until the frame is full.

In the scheduling of down-link packets, the packets are stored in the base station's buffer while awaiting transmission, so the FFA operates in a local buffer. However, in the scheduling of up-link packets, the buffer is distributed in the sense that it is composed of all the portables' buffers. In this case the base station has to receive updated information about the number of packets awaiting transmission in each portable buffer, so that the distributed buffers can be operated in conjunction as if they were a single buffer.

The FFA serves the packets according to their order of arrival in the buffer. However, this algorithm could be modified in order to serve packets according to some policy that privileges real time services. See [8] for example.

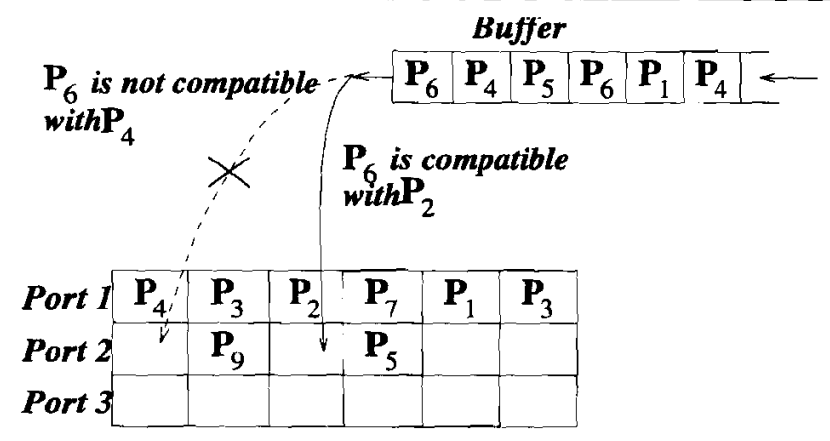

TS 1 TS 2 TS 3 TS 4 TS 5 TS 6 TS: Time Slo

Figure 11: The First Fit Algorithm.

It has been assumed that the base station keeps updated information about the compatibility condition among the portables and about the number of packets awaiting transmission in each buffer. In [3], a frame structure with overheads that allow the base station to acquire and update this information is proposed.

\section{FFA SIMULATION}

Simulations were run in order to evaluate the FFA performance in location 1. Only up-link traffic was simulated, and performance was evaluated with and without power control. The traffic model used in these simulations was a well behaved VBR service in which a new packet for portable $i$ $(i=1,2, \ldots M)$ arrives in its buffer during a time slot according to the Bernoulli process. It was assumed that the base station had immediate knowledge of a packet arrival.

The simulation results can be seen in Figure 12. These results were obtained assuming a capture threshold of $10 \mathrm{~dB}$ and a frame size of 20 time slots. However, it was shown in [3] that the maximum throughput is not dependent on the size of the frame though it affects the latency of the packets, so a small frame size is desirable. The curves are for mean buffer occupancy and throughput as functions of the system load, which is the traffic generated by all the $M$ portables, where each portable contributes $\frac{1}{N}$ of the total load. Throughput is defined as the average number of packets transported per time slot. From the graphs we can observe that the throughput is equal to the load up to a point where it saturates. At this point the system reaches its maximum throughput, and thus the mean buffer occupancy rises rapidly.

In location 1, the maximum throughput with three ports was around 2.1 without power control and around 2.5 with power control. The results obtained with four ports (not plotted) were not significantly better than those obtained with three ports. The reason can be seen in figure 6 , where the value of the average compatibility among four portables $(A C 4)$ is practically zero at a capture threshold of $10 \mathrm{~dB}$. However, we should note that the 3-port case with power control was able to provide a maximum throughput of 2.5 , even though the average compatibility among three portables $(A C 3)$ is only 0.04 at a capture threshold of $10 \mathrm{~dB}$ (see FIG. 6).

Figure 12 shows the FFA maximum throughputs (the 

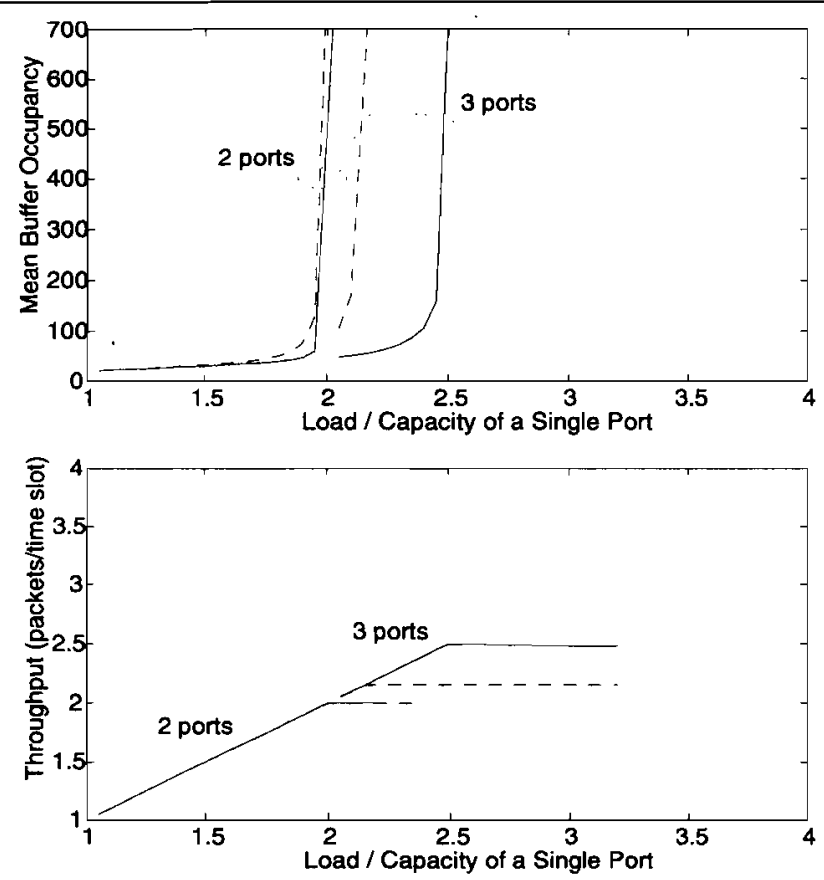

Figure 12: FFA performance for up-link traffic in location 1 with power control (solid lines) and without power control (dashed lines) assuming a capture threshold of $10 \mathrm{~dB}$.

points where the curves saturated) for a capture threshold of $10 \mathrm{~dB}$. In [3], the measurement results were analyzed in terms of the FFA maximum throughput as a function of the capture threshold. For example, Figure 13 shows the up-link maximum throughput that can be achieved in location 1 with different capture threshold values. However, due to a feature of the measuring experiment, the results in Figure 13 are valid only for a sectorization level of ten. In the following section, we describe a statistical model for indoor multipath propagation which can be used to investigate how the sectorization level affects the average compatibility among the portables and, consequently, how it affects the maximum throughput in the microcells. Intuitively, we can expect that the use of a higher sectorization level, which translates into using antennas with better directivity, can improve the average compatibility among the terminals, since antennas with better directivity can filter out more of the interfering multipaths.

The problem was investigated through Monte Carlo simulations of an indoor multipath propagation statistical model. This was preferred over two other options: simulation by means of ray tracing [12][13] and actual measurements using antennas of different beamwidths.

The main difficulty related to ray tracing is that this technique requires a detailed description of the indoor layout and a knowledge of reflection and transmission coefficients of walls, doors, windows, etc. We also had the option of measuring indoor locations using antennas of different beamwidths. However, we considered this approach too complicated because each sectorization level would require its own directive antenna, and so the measurements would have to be repeated for each sectorization level investigated. Moreover, these two options have the drawback of being ad hoc, unlike an indoor multipath propagation statistical model that can be applied to

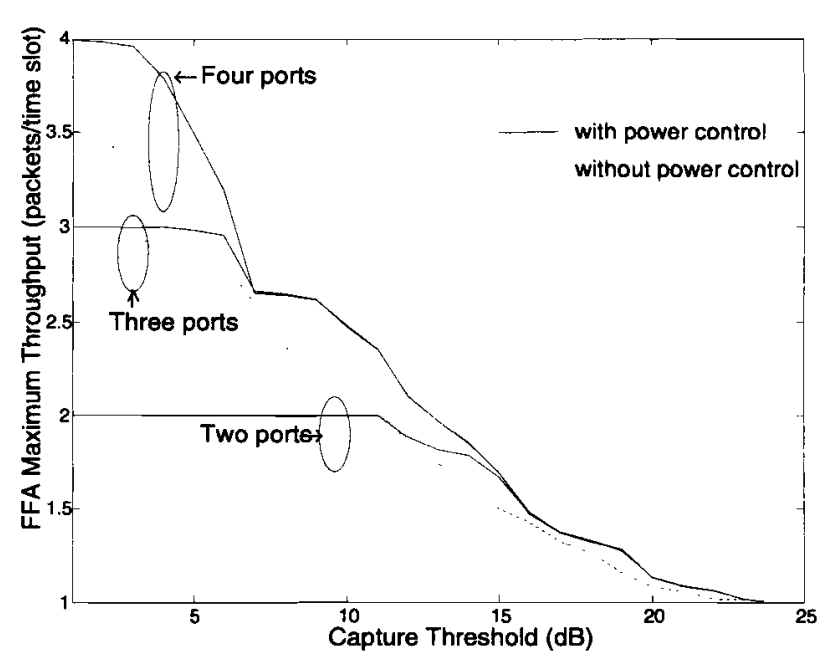

Figure 13: FFA up-link maximum throughput in location 1.

any location provided that some parameters in the model are adjusted appropriately.

\section{STATISTICAL MODEL DE- SCRIPTION}

In this section, we describe a statistical model for indoor propagation. The model is proposed by Spencer et al. [9] as an extension of the Saleh-Valenzuela model [11], and it accounts for amplitude, time and angle of ray arrival. The statistical model for indoor multipath propagation proposed by Saleh and Valenzuela does not take into consideration the angles of arrival of the multipaths, which are necessary in our investigation. However, it has served as the starting point for a statistical model developed by Spencer et al. that incorporates the angle of arrival into multipath propagation. The resulting model is a combination of two statistically independent processes, one governing ray amplitudes and arrival times, and the other governing ray angles of arrival.

\section{Modeling Ray Amplitude and Ray Arrival Time}

This part of the model is actually the Saleh-Valenzuela model itself. It assumes that, in the time axis, rays arrive in clusters. The cluster arrival time ( $T_{l}$, see Figure 14), which is defined as the arrival time of the first ray of the cluster, is modeled by a Poisson arrival process with rate $\Lambda$. A Poisson arrival process is also used, but with rate $\lambda$, to model ray arrival times ( $\tau_{k l}$, see figure 14 ) within a cluster. The distributions of cluster and ray arrival times are given respectively by

$$
p\left(T_{l} \mid T_{l-1}\right)=\Lambda e^{-\Lambda\left(T_{l}-T_{l-1}\right)}
$$

and

$$
p\left(\tau_{k l l} \mid \tau_{(k-1) l}\right)=\lambda e^{-\lambda\left(\tau_{k l}-\tau_{(h-1, l)}\right)},
$$

where $l$ represents the $l$-th cluster and $k l$ represents the $k$-th ray within the $l$-th cluster. 


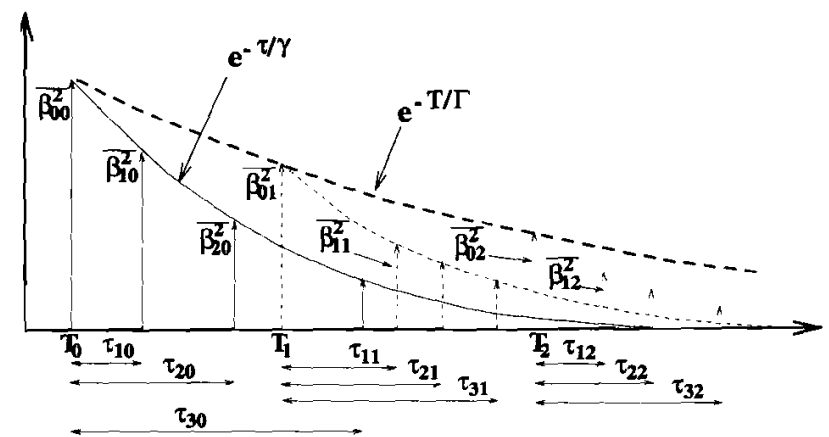

Figure 14: A representation of the clustering phenomenon in multipath propagation.

Let us assume that the $k l$-th ray has amplitude $\beta_{k l}$ and phase $\phi_{k l l}$. Therefore the channel impulse response is given by

$$
h(t)=\sum_{l=0}^{\infty} \sum_{k=0}^{\infty} \beta_{k l} e^{-j \phi_{k l}} \delta\left(t-T_{l}-\tau_{k l}\right) .
$$

In this model, $\beta_{k l}$ is a Rayleigh distributed random variable whose mean square value is illustrated in figure 14 , and is expressed mathematically by a double-exponential decay

$$
\overline{\beta_{k l}^{2}}=\overline{\beta^{2}(0,0)} e^{-T_{l} / \Gamma} e^{-\tau_{k l} / \gamma}
$$

where $\overline{\beta^{2}(0,0)}$ is the average power of the first arrival, and $\Gamma$ and $\gamma$ are cluster and ray power-decay time constants, respectively. The following expression for $\overline{\beta^{2}(0,0)}$ is derived by Saleh and Valenzuela [11]:

$$
\overline{\beta^{2}(0,0)} \approx \frac{G(1 m) r^{-\alpha}}{\gamma \lambda}
$$

where $r$ is the separation distance of the transmitter and receiver, $G(1 \mathrm{~m})$ is the channel gain at a distance of 1 meter $(r=1)$, and $\alpha$ is the channel loss parameter which depends on the characteristics of the indoor environment. A typical value of $\alpha$ for office buildings is 3 [11], but values as high as 6 have been reported for office buildings with metalized partitions [14].

In [9] and [11], $T_{0}$, the arrival time of the first cluster, is defined in different ways. We will assume the definition given in [9], which is

$$
T_{0}=\frac{r}{c}
$$

where $\mathrm{c}$ is the speed of light.

\section{Modeling the Ray Angle of Arrival}

In their measurements, Spencer et al. could not observe any correlation between the angle and the time of ray arrival Based on this fact, they propose a statistical model for ray angle of arrival that is independent on the ray arrival time. The following expression represents the proposed angular impulse response of the multipath channel:

$$
h(\theta)=\sum_{l=0}^{\infty} \sum_{l=0}^{\infty} \beta_{k l} \delta\left(\theta-\Theta_{l}-\omega_{k l}\right),
$$

\begin{tabular}{|l|r|r|}
\hline Parameter & Building 1 & Building 2 \\
\hline$\Gamma$ & $33.6 \mathrm{~ns}$ & $78.0 \mathrm{~ns}$ \\
$\gamma$ & $28.6 \mathrm{~ns}$ & $82.2 \mathrm{~ns}$ \\
$1 / \Lambda$ & $16.8 \mathrm{~ns}$ & $17.3 \mathrm{~ns}$ \\
$1 / \lambda$ & $5.1 \mathrm{~ns}$ & $6.6 \mathrm{~ns}$ \\
$\sigma$ & $25.5^{\circ}$ & $21.5^{\circ}$ \\
\hline
\end{tabular}

Table 1: Parameters estimated by Spencer et al.

where $\beta_{k l}$ has the same meaning as in Equation $11 ; \theta_{l}$ is the mean angle of the $l$-th cluster, that is, the mean angle of the rays arriving within the $l$-th cluster; and $\omega_{k l}$ is the angle deviation from $\Theta_{l}$ making the $k l$-th ray arrive at an angle of $\Theta_{l}+\omega_{k l}$.

It is proposed in [9] that $\Theta_{l}$ be uniformly distributed throughout the interval $[0,2 \pi)$ and that the ray angle deviation, $\omega_{k l}$, be modeled as a zero mean Laplacian distribution with standard deviation $\sigma$ :

$$
p(\omega)=\frac{1}{\sqrt{2} \sigma} e^{-|\sqrt{2} \omega / \sigma|} .
$$

\section{Estimated Parameters}

The resulting statistical model, which accounts for amplitude, time, and angle of arrival, is defined by five parameters: $\Lambda, \lambda$, $\Gamma, \gamma$, and $\sigma$. Table 1 shows Spencer et al.'s estimations for these parameters, which were based on measurements taken in two different buildings and reported in [10]. The frequency used in these measurements was $7 \mathrm{GHz}$, which is relatively close to the $5.8 \mathrm{GHz}$ frequency that we used in our measurements [3].

In order to understand why the parameter values are building-dependent, we need a physical interpretation of the model. Spencer et al. observed that the strongest cluster was almost always associated with a direct line of sight path, even when this line was blocked by walls. Weaker clusters were apparently caused by back wall reflections and doorway openings. Therefore the formation of clusters is related to building superstructures. The rays within a cluster are related to smaller objects, such as furniture, that are in the vicinity of the transmitter and the receiver

\section{AVERAGE COMPATIBILITY SIMULATIONS}

This section shows how the statistical model described in the previous section can be used to simulate average compatibility among portables. We first define the dimensions of the indoor location. Here, we assume an area of $25 \times 30 \mathrm{~m}^{2}$, which is the area of our indoor location 1 (see Figure 5). In order to compose a simulation scenario, we place the base station at the center of this area, and we place $M$ portables (we will be considering $M=50$ ) at random positions uniformly distributed within the area. This is illustrated in Figure 15.

Next, the steps in the following list are repeated in order to generate a total of four clusters and 15 rays per cluster for 


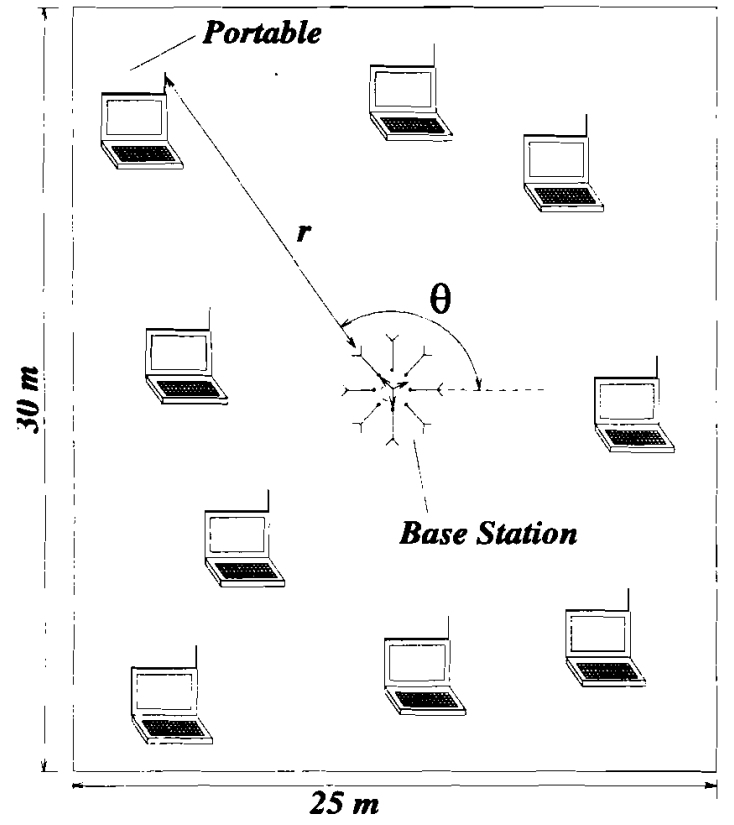

Figure 15: A generic indoor location of $25 \times 30 \mathrm{~m}^{2}$.

each portable. These numbers were chosen based on the observation that using more than four clusters and more than 15 rays per clusters did not alter the simulation results significantly.

- Portable-Base distance $r$ is used in (14) to compute the arrival time of the first cluster $T_{0}$.

- The probability distributions of (9) and (10) are used to draw the cluster arrival times $\left(T_{l}\right)$ and the ray arrival times $\left(\tau_{k l}\right)$, respectively.

- Portable-Base distance $r$ is used in (13) to compute the average power of the first arrival $\left(\overline{\beta_{00}^{2}}\right)$.

- Cluster arrival time, ray arrival times and the average power of the first arrival are used in (12) to compute the mean square value of the ray amplitudes $\left(\overline{\beta_{k l}^{2}}\right)$.

- A Rayleigh distribution with mean square values of $\overline{\beta_{k l}^{2}}$ is used to draw the $k l$-th ray amplitude $\left(\beta_{k l}\right)$.

- A random phase $\phi_{k i l}$ is drawn for each ray using uniform distribution in the interval $[0,2 \pi)$. Therefore the channel impulse response for the $k l$-th path is given by $\beta_{k, l} e^{-j)_{h l}} \delta\left(t-T_{l}-\tau_{k l l}\right)$.

- The angle of the line of sight path $\Theta$ (see figure 15) is used as angle of arrival for the first cluster $\Theta_{0}$. This is based on the observation made by Spencer et al. that the strongest cluster was almost always associated with a direct line of sight path, even when this line was blocked by walls.

- A random angle of arrival $\Theta_{l}$ is drawn for each of the three remaining clusters using a uniform distribution in the interval $[0,2 \pi)$. Note that this does not incorporate possible angular correlations among clusters of neighboring portables. figure 16 illustrates why cluster arrival angles of neighboring portables are likely to be correlated. We investigated the effect of these correlations, and concluded that they cause only a slight decrease in the average compatibilities, so they can be disregarded.

- The probability distribution of (16) is used to draw the angle of arrival deviation $\omega_{k l}$ for each path. Therefore the ray angle of arrival of the $k l$-th ray is $\Theta_{l}+\omega_{k l}$.

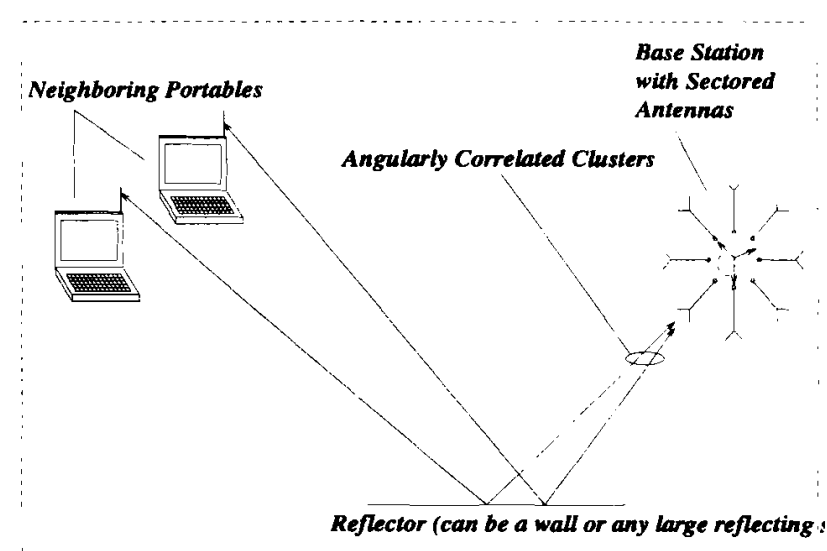

Figure 16: Illustration of angular correlation between clusters of neighboring portables.

\section{Computing Average Power}

Once we have obtained the angle of arrival and channel impulse response for each path, we can proceed to compute the average power received by the base station in each of its antenna sectors when a given portable transmits. In order to compute the average power received in each sector, we need the channel impulse response between the $i$-th sector and a given portable. Assuming four clusters and 15 rays per cluster, this can be expressed by

$$
h_{i}(t)=\sum_{l=0}^{3} \sum_{k=0}^{14} \beta_{k l} e^{-j \phi_{k l}} \delta\left(t-T_{l}-\tau_{k l}\right) g_{i}\left(\Theta_{l}+\omega_{k l}\right),
$$

where $g_{i}(\theta)$ is the $i$-th antenna sector gain at angle $\theta$.

Now we are ready to compute the average power received in each sector when a given portable transmits. We are interested in the average power received in a bandwidth of $20 \mathrm{MHz}$ centered at $5.8 \mathrm{GHz}$ which represents the setup of the measuring experiment described in [3]. This can be expressed as

$$
G_{i}=\frac{1}{20 \times 10^{6}} \int_{-10 \times 10^{\prime i}}^{10 \times 10^{\prime \prime}}\left|H_{i}\left(f-5.8 \times 10^{9}\right)\right|^{2} d f,
$$

where $H_{i}(f)$ is the channel frequency response between the portable and the $i$-th sector which is the Fourier transform of $h_{i}(t)$ :

$$
H_{i}(f)=\int_{-\infty}^{\infty} h_{i}(t) e^{-j 2 \pi f t} d t .
$$

Substituting (17) into (19) we obtain

$$
H_{i}(f)=\sum_{l=0}^{3} \sum_{k=0}^{14} g_{i}\left(\Theta_{l}+\omega_{k l}\right) \beta_{k l} e^{-j \phi_{k l}} e^{-j 2 \pi f\left(T_{i}+\tau_{h l}\right)} \text {. }
$$




\section{Aleandro S. Macedo and Elvino S. Sousa Broadband Indoor Wireless System with In-Cell Frequency Reuse Based on Sectored Antennas}

We can use (18) to compute the average power received in the $i$-th antenna sector when portable $p$ transmits. Let us rename it $G_{p, i}$; it can also be seen as the average power received by portable $p$ when the base station transmits through its $i$-th antenna sector. This is true because up-link and downlink transmissions occur in the same frequency band, therefore there is up-link/down-link channel reciprocity. Consider antenna sector $B(p)$ which provides the best average power to portable $p$. Therefore the best average power for portable $p$ is $G_{p, B(p)}$. In order to verify compatibility among portables, we use these values in the conditions defined in (3) and (4) for the down-link case and for the up-link case, respectively.

\section{RESULTS}

The steps described in the previous section were followed to simulate average compatibilities among portables uniformly distributed in a $25 \times 30 \mathrm{~m}^{2}$ indoor location. The results were averaged over ten different scenarios, so that, for a given set of parameters, the simulations were run ten times, and a different draw of portables positions was used for each run. The results were then presented as the average of these ten outputs. We chose to simulate only down-link average compatibilities since, as we saw in Section 5, up-link average compatibilities are close matches to down-link average compatibilities when power control is used.

The first simulation used the parameter values in Table 1 for building 1 which are: $\Gamma=33.6 \mathrm{~ns}, \gamma=28.6 \mathrm{~ns}, 1 / \Lambda=$ $16.8 \mathrm{~ns}, 1 / \lambda=5.1 \mathrm{~ns}$, and $\sigma=25.5^{\circ}$. The channel loss parameter used was $\alpha=3$. However, changing the value of $\alpha$ did not affect the simulation results, as expected for the down-link case.

We have to decide on the number of sectors to simulate. Let us start with a sectorization level of 10 and assume that each sector employs a horn antenna like the one which was used for the measurements reported in Section 5. This antenna has approximately $36^{\circ}$ of $3 \mathrm{~dB}$ beamwidth and its radiation pattern is shown in Figure 17.
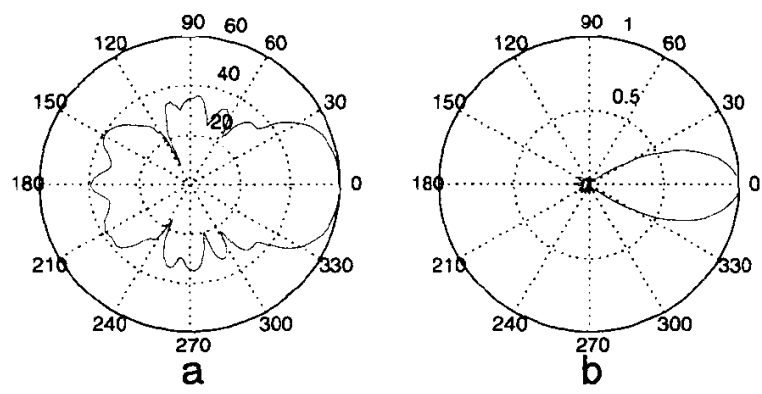

Figure 17: (a) Polar coordinate plots of power radiation pattern on logarithmic scale; and (b) normalized field strength pattern on linear scale of the horn antenna used in the experiment. Note: the plotted values were normalized by making the maximum value equal to $60 \mathrm{~dB}$ in the logarithmic plot, and by making the maximum value equal to 1.0 in the linear plot.

figure 18 shows the simulation results compared with the average compatibilities measured in location 1 . We can see that the measured and the simulated results did not match very well. However, the matching can be improved by adjusting the simulation parameters as follows.

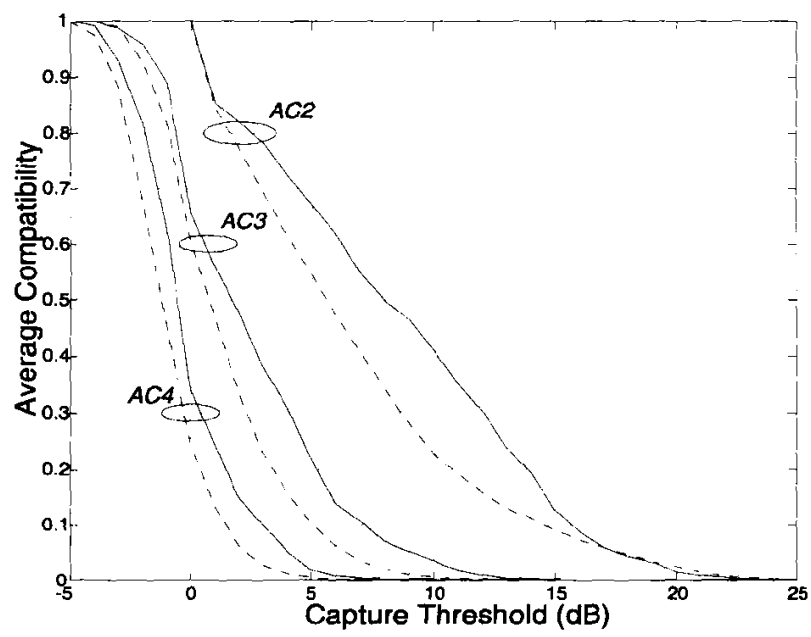

Figure 18: Average compatibilities simulated with parameters of building 1 (dashed lines) compared with average compatibilities measured in location 1 (solid lines).

In Table 1, the main differences between the estimates of the two buildings are the values of $\Gamma$ and $\gamma$. The lower values of $\Gamma$ and $\gamma$ in building 1 arise because there is greater attenuation with internal walls of cinder blocks than in building 2 whose internal walls are gypsum board [10]. Therefore, we can expect location 1 (see FIG. 5), whose internal walls are concrete blocks, to attenuate more than building 1. For example, we simulated average compatibilities with $\Gamma=10 \mathrm{~ns}$ and $\gamma=10 \mathrm{~ns}$, and compared them with the average compatibilities of location 1. The results are shown in figure 19. We can see that matching was improved at low values of the capture threshold, but the mismatch worsened at higher capture threshold values. This made us think that, while taking measurements in location 1 , the horn antenna radiation pattern may have been distorted due to the proximity of the measuring equipment and metal structures on the ceiling (light fixtures). The most likely form of distortion is enhancement of the back and side lobes, which may have been caused by rays that were reflected into the main lobe by the equipment and/or by the light fixtures. In order to investigate this possibility, we ran a simulation using the antenna radiation pattern of the horn antenna distorted by back/side lobes in each sector. We added three $-15 \mathrm{~dB}$ back/side lobes to the original antenna pattern. The resulting empirical antenna pattern is shown in Figure 20.

Figure 21 shows the average compatibilities simulated with this distorted antenna pattern compared with the average compatibilities measured in location 1 . As the curves matched very well, we concluded that antenna back/side lobes are responsible for the loss in average compatibility at the high capture threshold values observed in Figure 19. Therefore, antennas with small side/back lobes in their radiation patterns should be used to prevent this from happening. Also, metal structures, such as light fixtures should be kept as 


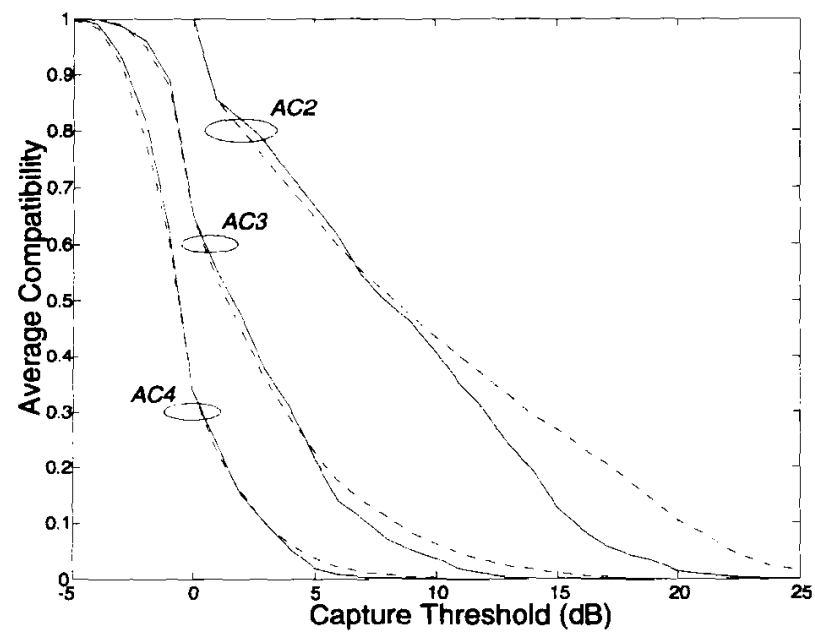

Figure 19: Average compatibilities simulated with $\Gamma=10$ ns and $\gamma=10 \mathrm{~ns}$ (dashed lines) compared with average compatibilities measured in location 1 (solid lines).
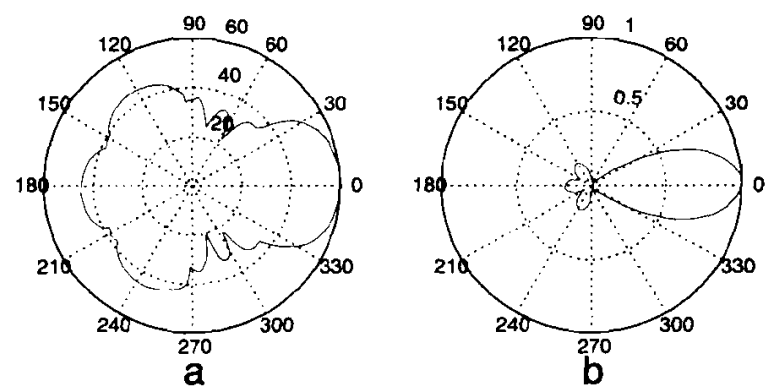

Figure 20: (a) Polar coordinate plots of distorted power radiation pattern on logarithmic scale; and (b) distorted field strength pattern on linear scale of the horn antenna used in the experiment.

far as possible from the antennas to avoid distorting their radiation patterns. However this is important only if the system is to be operated at high values of the capture threshold.

\section{A Model for Generating Antenna Patterns}

So far, our simulations have been based on the antenna pattern of the horn antenna used for the indoor measurements reported in [3]. For the following simulations, we will generate antenna patterns using a model where the antenna beamwidth is a variable. With this model, the $i$-th antenna sector normalized field strength radiation pattern can be generated as

$$
g_{i}(\theta)= \begin{cases}\frac{\sin \left\{\frac{2.78[\theta-(i-1) \Phi]}{\Phi}\right\}}{\frac{2.78[\theta-(i-1) \Phi]}{\Phi}} & \text { for antenna main lobe } \\ a_{0} & \text { otherwise, }\end{cases}
$$

where $a_{0}$ is a uniform side lobe which we assume to be $30 \mathrm{~dB}$ below the main beam gain, and $\Phi$ is the $3 \mathrm{~dB}$ beamwidth of the antenna. The formula used in (21) to obtain the main lobe of the antenna resulted from an approximation to the formula for unidirectional radiation from uniform aperture distribu-

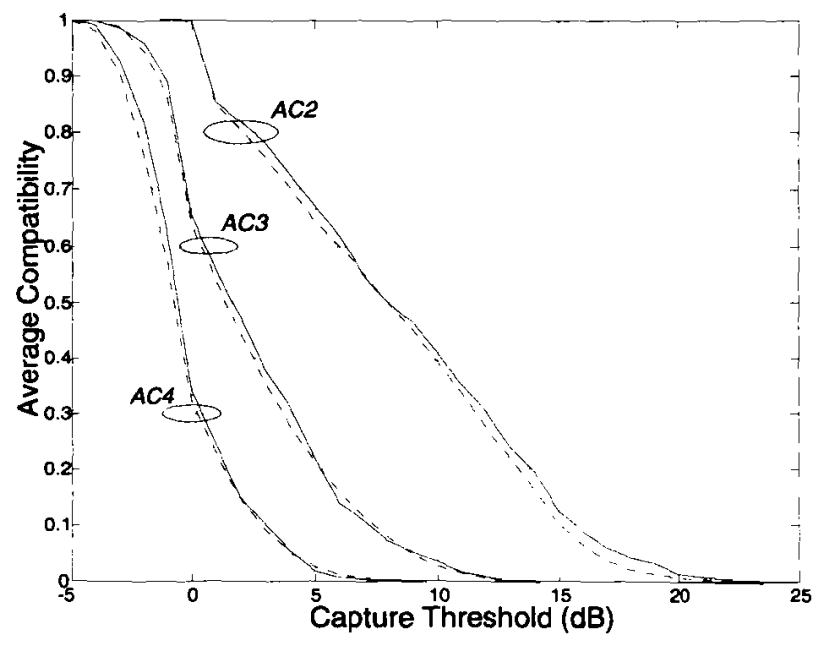

Figure 21: Average compatibilities simulated with distorted antenna pattern (dashed lines) compared with average compatibilities measured in location 1 (solid lines).

tion (see [15]-p.517).

For example, Figure 22 shows the pattern for antenna sector $1(i=1)$ with $\Phi=36^{\circ}$ superimposed on the pattern of the horn antenna which we have used in the measurements.
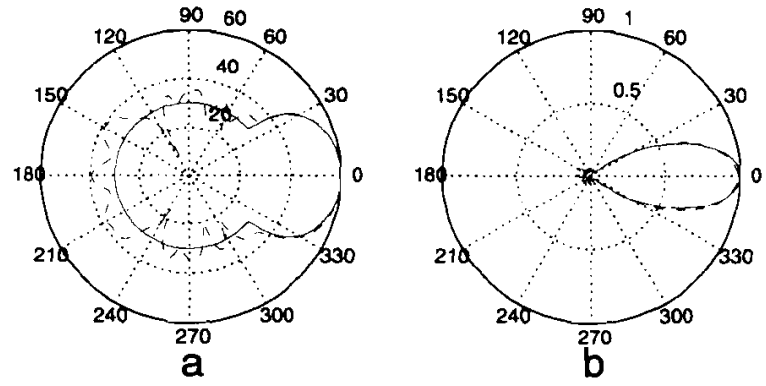

Figure 22: Radiation patterns on logarithmic scale (a) and on linear scale (b) of $36^{\circ}$ beamwidth antenna (solid) and horn antenna (dashed).

With this antenna pattern model we can simulate average compatibilities with a generic sectored antenna system with $N_{s}$ sectors using $N_{s}$ antennas with $\Phi=360^{\circ} / N_{s}$. For example, Figure 23 shows sectored antenna systems with $3,5,10$ and 20 antennas.

\section{FFA Maximum Throughput as a Function of Sectorization Level}

Figure 24 shows the FFA maximum throughput obtained with sectorization levels of $4,10,20$ and 50 sectors, and with $\Gamma=$ $10.0 \mathrm{~ns}, \gamma=10.0 \mathrm{~ns}, 1 / \Lambda=16.8 \mathrm{~ns}, 1 / \lambda=5.1 \mathrm{~ns}$ and $\sigma=25.5^{\circ}$. The number of ports used to obtain the results were $3,5,7$ and 11 when using $4,10,20$ and 50 sectors, respectively.

These results show that there is considerable potential for increasing the maximum throughput by increasing the number of sectors in the microcell. The limit to this capacity gain 

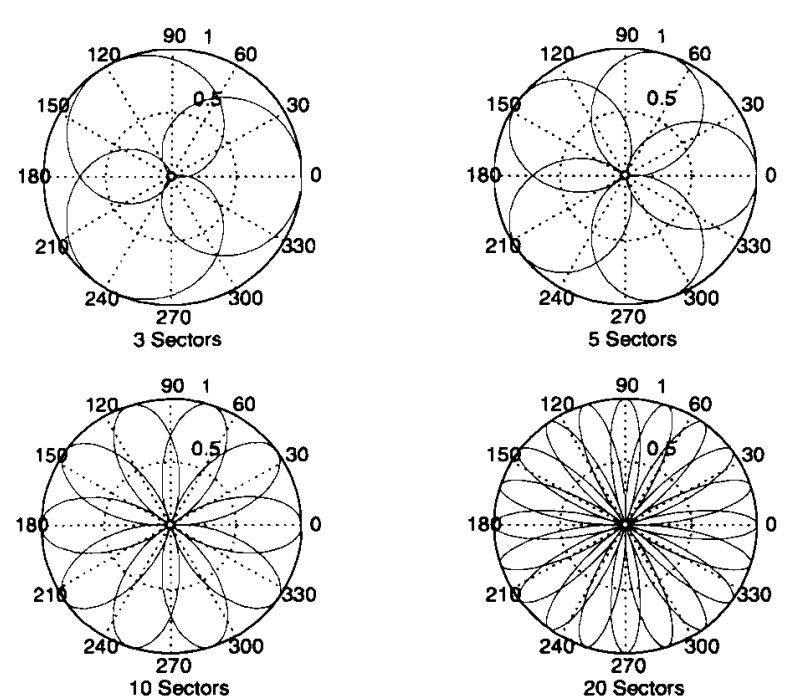

Figure 23: Sectored antenna systems with $3,5,10$ and 20 antennas.

seems to be related to the complexity of the sectored antenna system, since it is not obvious to us that, for example, a 50sector antenna system built to operate at a frequency of 5.8 $\mathrm{GHz}$ can be made compact enough to be installed indoors.

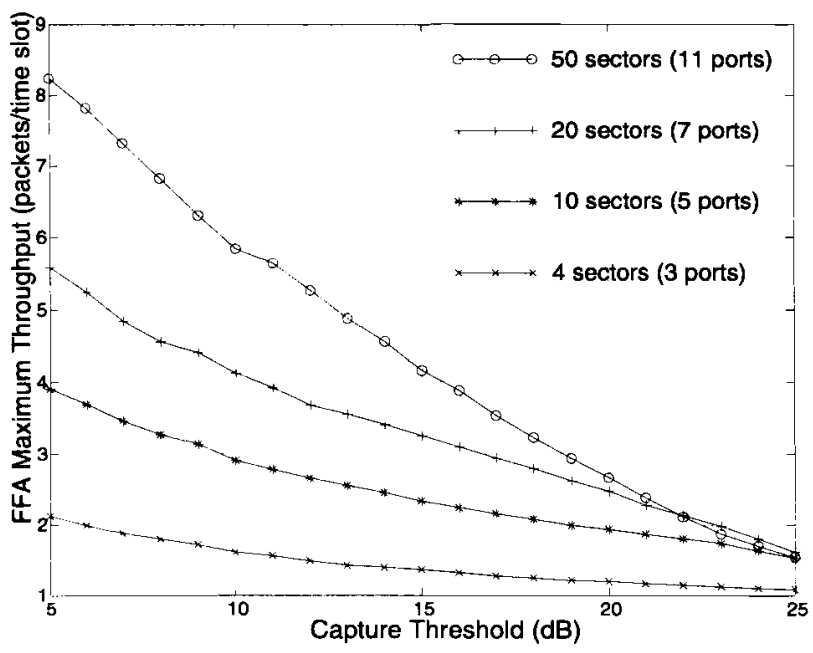

Figure 24: FFA maximum throughput with 4, 10, 20 and 50 sectors.

\section{CONCLUSIONS}

This paper investigated a new indoor communication system in which a base station uses sectored antennas, and portable terminals use omni-directional antennas. The system is able to handle simultaneous packet transmissions in a microcell.

The compatibility concept was proposed, and experimental results of average compatibility were obtained assuming sectorazation level of ten in a closed indoor environment represented by a section of a building floor.

A multiple access scheme that is able to take advantage of the compatibilities among portables in order to transmit more than one data packet per time slot was proposed. The FFA algorithm was proposed for scheduling simultaneous packet transmissions in the microcell.

A statistical model of indoor multipath propagation was used to investigate the performance of the proposed system with different sectorization levels. The simulation parameters of this model were adjusted to fit the experimental results obtained with sectorization level ten. The simulations showed that there is considerable potential for increasing the maximum throughput by increasing the number of sectors in the microcell.

\section{REFERENCES}

[1] D. Buchholz, P. Odlyzko, M. Taylor and R. White, "Wireless In-Building Network Architecture and Protocols," IEEE Network Magazine, Vol. 5, No. 6, pp. 31-38, Nov. 1991.

[2] A. S. Mahmoud, D. D. Falconer and S. A. Mahmoud, "A Multiple Access Scheme For Wireless Access to a Broadband ATM LAN Based on Polling and Sectored Antennas," PIMRC'95, Vol. 3, pp. 1047-1051, Toronto Canada, Sept. 1995.

[3] A. S. Macedo and E. S. Sousa, "In-Cell Frequency Reuse for Broadband Indoor Wireless Systems Using Sectored Antennas," To appear in Wireless Personal Communications, Special Issue on Wireless Broadband Communications.

[4] J. E. Mitzlaff, "Radio Propagation and Anti-Multipath Techniques in the WIN Environment," IEEE Network Magazine, Vol. 5, No. 6, pp. 21-26, Nov. 1991.

[5] FCC 96-193 Document, Released on May 7, 1996.

[6] M. R. Garey and D. S. Johnson, Computers and Intractability - A Guide to the Theory of NP. Completeness, San Francisco: W. H. Freeman, 1979.

[7] M. de Prycker, Asynchronous Transfer Mode: Solution for Broadband ISDN, New York: Ellis Horwood, 1991.

[8] N. Movahhedinia, G. Stamatelos and H. M. Hafez, "Polling-Based Multiple Access for Indoor Broadband Wireless Systems," PIMRC'95, Vol. 3, pp. 1052-1056, Toronto Canada, Sept. 1995.

[9] Q. Spencer, M. Rice, B. Jeffs and M. Jensen, "A Statistical Model for Angle of Arrival in Indoor Multipath Propagation,' VTC'97, Vol. 3, pp. 1415-1419, Phoenix, Arizona, USA, May 1997.

[10] Q. Spencer, M. Rice, B. Jeffs and M. Jensen, "Indoor Wideband Time/Angle of Arrival Multipath Propagation Results," VTC'97, Vol. 3, pp. 1410-1414, Phoenix, Arizona, USA, May 1997.

[11] A. A. M. Saleh and R. A. Valenzuela, "A Statistical Model for Indoor Multipath Propagation," IEEE Journal on Selected Areas in Communications, Vol. SAC-5, No. 2, pp. 128-137, February 1987. 
[12] G. Yang, K. Pahlavan and T. Holt, "Sector Antenna and DFE Modems for High Speed Indoor Radio Communications," IEEE Transactions on Vehicular Technology, Vol. 43, No. 4, pp. 925-933, Nov. 1994.

[13] J. McKown and R. Lee Hamilton, Jr," "Ray Tracing as a Design Tool for Radio Networks," IEEE Network Magazine, Vol. 5, No. 6, pp. 27-30, Nov. 1991.

[14] S. E. Alexander, "Characterizing Buildings for Propagation at $900 \mathrm{MHz}$," Electron Lett., Vol. 19, No. 20, pp. 860, Sept. 1983.

[15] J. D. Kraus, Antennas, 2nd ed., New York: Mc GrawHill, 1988.

Aleandro S. Macedo was born in Minas Gerais, Brazil in 1966. He received the B.S. degree from the Federal University of Minas Gerais, Brazil, in 1990; the M.A.Sc. degree from the State University of Campinas - Brazil, in 1993; and the Ph.D. degree from the University of Toronto in 1998; all in electrical engineering. He has performed research in the area of architecture design of SDH/SONET equipment. His current interests are in the areas of wireless communications, indoor channel measurements/modeling and multicarrier modulation.

Elvino S. Sousa received the B.A.Sc. degree in engineering science, and the M.A.Sc. degree in electrical engineering from the University of Toronto in 1980 and 1982 respectively, and the Ph.D. degree in electrical engineering from the University of Southern California in 1985. Since 1986 he has been with the department of Electrical and Computer Engineering at the University of Toronto where he is presently a Professor and Chair of the Communications Group. From 1986 to 1996 he was a Natural Sciences and Engineering Research Council of Canada (NSERC) University Research Fellow. He has performed research in the areas of packet radio networks and spread spectrum systems since 1983 . His current interests are in the areas of spread spectrum systems, mobile communications, indoor wireless communications, and wireless LAN's. At the University of Toronto he teaches courses in mobile communications and error control codes. Currently he has research projects on the performance of microcellular networks, wireless multimedia networks, and wireless LAN's. He has given many short courses and lectures on the topics of spread spectrum and wireless communications in a number of different countries. He is an associate editor in the area of CDMA systems for the IEEE Transactions on Communications, and was the technical program chairman for PIMRC' 95. 